$\underline{\text { Review Article }}$

\title{
REVIEW ARTICLE: SOLUBILITY ENHANCEMENT BY SOLID DISPERSION
}

\author{
A. N. PATIL*, D. M. SHINKAR, R. B. SAUDAGAR
}

Department of Pharmaceutics, R. G. Sapkal College of Pharmacy Anjaneri, Nashik 422213, Maharashtra, India

Email: patilajit36@gmail.com

Received: 28 Dec 2016, Revised and Accepted: 20 Mar 2017

\begin{abstract}
Keywords: Solid dispersion, Solubility enhancement DOI: http://dx.doi.org/10.22159/ijcpr.2017v9i3.19583

\section{INTRODUCTION}

Solid dispersion (SD) has been widely used to improve the dissolution rate, solubility, and oral absorption of poorly water-soluble drugs. SD refers to the group of solid products consisting of at least two different components, generally a hydrophilic matrix and a hydrophobic drug: the matrix can be either crystalline or amorphous [1]. The solid dispersion was first introduced to overcome the low bioavailability of
\end{abstract}

Enhancement of solubility, dissolution rate and bioavailability of the drug is a very challenging task in drug development, nearly $40 \%$ of the new chemical entities currently being discovered are poorly water soluble drugs. The solubility behaviour of the drugs remains one of the most challenging aspects in formulation development. This results in important products not reaching the market or not achieving their full potential. Solid dispersion is one of the techniques adopted for the formulation of such drugs and various methods are used for the preparation of solid dispersion. Solid dispersion is generally prepared with a drug which is having poor aqueous solubility and hydrophilic carrier. This article review various methods and concept of solid dispersion, criteria for drug selection, advantage and disadvantage, characterization, and application.

(C) 2017 The Authors. Published by Innovare Academic Sciences Pvt Ltd. This is an open access article under the CC BY license (http://creativecommons.org/licenses/by/4.0/]

lipophilic drugs by forming a eutectic mixture of drugs with water soluble carriers. Approximate $40 \%$ of new chemical entities (NCE) being synthesized by combinatorial screening programs possessing superior pharmacological activities are poorly soluble, which is a great obstacle in formulation development [2]. Biopharmaceutical classification system (BCS) highlights the dissolution as rate limiting step for oral absorption of BCS class 2 and class 4 drugs. BCS class 2 and class 4 drugs have low solubility.

Table 1: Solubility criteria as per USP and BP

\begin{tabular}{ll}
\hline Descriptive term & Part of solvent required per part of solute \\
\hline Very Soluble & Less than 1 \\
Freely Soluble & From 1 to 10 \\
Soluble & From 10 to 30 \\
Sparingly Soluble & From 30 to 100 \\
Slightly Soluble & From 100 to 1000 \\
Very Slightly Soluble & From 1000 to 10000 \\
Practically insoluble & 10,000 and over \\
\hline
\end{tabular}

Table 2: Type of solubility enhancement techniques [9, 10]

\begin{tabular}{llll}
\hline S. No. & Chemical modification & Physical modification & Other \\
\hline 1 & Salt Formation & Particle size reduction & Supercritical fluid method \\
2 & Co-crystallization & Modification of the crystal habit & Spray freezing into liquid and Lyophillization \\
3 & Co-solvency & Complexation & Evaporative precipitation into aqueous solution \\
4 & Hydrotropic & Solubilization by surfactants & Solvent evaporation method \\
5 & Solubilizing agent & Drug dispersion in carriers & Hot melt extrusion \\
& & Solid solution & \\
& & Eutectic mixtures & \\
6 & Nanotechnology & Solid dispersion & Electrostatic spinning method \\
7 & - & - & Direct capsule filling \\
8 & - & - & Polymeric Alteration \\
9 & - & - & Lyophilization technique \\
\hline
\end{tabular}

Types of solid dispersions

Eutectic mixtures [2, 3]

A simple eutectic mixture consists of two compounds which are completely miscible in the liquid state but only to a very limited extent in the solid state. It is prepared by rapid solidification of fused melt of two components that show complete liquid miscibility but negligible solid-solid solution

Amorphous precipitation in crystalline matrix $[4,5]$

This is similar to simple eutectic mixtures but only difference is that drug is precipitated out in an amorphous form. 


\section{Solid solution [6]}

Solid solutions are comparable to liquid solutions, consisting of just one phase irrespective of the number of components. In the case of solid solutions, the drug's particle size has been reduced to its absolute minimum viz. the molecular dimensions and the dissolution rate is determined by the dissolution rate of the carrier. Classified according to their miscibility (continuous versus discontinuous solid solutions) or second, according to the way in which the solvate molecules are distributed in the solvendum (substitutional, interstitial or amorphous).

\section{Continuous solid solutions}

In a continuous solid solution, the components are miscible in all proportions. Theoretically, this means that the bonding strength between the two components is stronger than the bonding strength between the molecules of each of the individual components. Solid solutions of this type have not been reported in the pharmaceutical world till date.

\section{Discontinuous solid solutions [6]}

In the case of discontinuous solid solutions, the solubility of each of the components in the other component is limited. Due to practical considerations, it has been suggested by Goldberg et al. that the term 'solid solution' should only be applied when the mutual solubility of the two components exceeds $5 \%$.

\section{Substitutional solid dispersions [7]}

Substitution is only possible when the size of the solute molecules differs by less than $15 \%$ or so from that of the solvent molecules. Classical solid solutions have a crystalline structure, in which the solute molecules can either substitute for solvent molecules in the crystal lattice or fit into the interstices between the solvent molecules.

\section{Interstitial solid solutions $[15,16]$}

In interstitial solid solutions, the dissolved molecules occupy the interstitial spaces between the solvent molecules in the crystal lattice. Solute molecule diameter should be less than 0.59 times than that of solvent molecular diameter.

\section{Glass solution and suspensions}

Glass solutions are a homogeneous glassy system in which solute dissolves in glass carrier. Glass suspensions are a mixture in which precipitated particles are suspended in glass solvent. Lattice energy is much lower in glass solution and suspension.

\section{Methods of solid dispersion [17-19]}

\section{Melting method}

2. Solvent methods

3. Melting solvent method (melt evaporation)

4. Melt extrusion methods

5. Lyophilization techniques

6. Melt agglomerations Process

7. The use of surfactant

8. Electrospinning

9. Super Critical Fluid (Scf) technologies

\section{Melting method}

The melting or fusion method is the preparation of a physical mixture of a drug and a water-soluble carrier and heating it directly until it melted. The melted mixture is then solidified rapidly in an ice-bath under vigorous stirring. The final solid mass is crushed, pulverized and sieved. Appropriately this has undergone many modifications in pouring the homogenous melt in the form of a thin layer onto a ferrite plate or a stainless steel plate and cooled by flowing air or water on the opposite side of the plate. In addition, a super-saturation of a solute or drug in a system can often be obtained by quenching the melt rapidly from a high temperature. Under such conditions, the solute molecule is arrested in the solvent matrix by the instantaneous solidification process. The quenching technique gives a much finer dispersion of crystallites when used for simple eutectic mixtures. However many substances, drugs or carriers, may decompose during the fusion process which employs high temperature. It may also cause evaporation of volatile drug or volatile carrier during the fusion process at high temperature. Some of the means to overcome these problems could be heating the physical mixture in a sealed container or melting it under vacuum or in presence of inert gas like nitrogen to prevent oxidative degradation of drug or carrier.

\section{Solvent method}

In this method, the physical mixture of the drug and carrier is dissolved in a common solvent, which is evaporated until a clear, solvent free film is left. The film is further dried to constant weight. The main advantage of the solvent method is thermal decomposition of drugs or carriers can be prevented because of the relatively low temperatures required for the evaporation of organic solvents.

\section{Melting solvent method}

It involves preparation of solid dispersions by dissolving the drug in a suitable liquid solvent and then incorporating the solution directly into the melt of polyethylene glycol, which is then evaporated until a clear, solvent free film is left. The film is further dried to constant weight. The $5-10 \%(w / w)$ of liquid compounds can be incorporated into polyethylene glycol 6000 without significant loss of its solid property. It is possible that the selected solvent or dissolved drug may not be miscible with the melt of the polyethylene glycol.

\section{Melt extrusion method}

The drug/carrier mix is typically processed with a twin screw extruder. The drug/carrier mix is simultaneously melted, homogenized and then extruded and shaped as tablets, granules, pellets, sheets, sticks or powder. The intermediates can then be further processed into conventional tablets. An important advantage of the hot melt extrusion method is that the drug/carrier mix is only subjected to an elevated temperature for about $1 \mathrm{~min}$, which enables drugs that are somewhat thermolabile to be processed. Solid dispersion by this method is composed of active ingredient and carrier, and prepare by hotstage extrusion using a corotating twin screw extruder. The concentration of the drug in the dispersions is always $40 \%(\mathrm{w} / \mathrm{w})$. The screw configuration consist of two mixing zones and three transport zones distributed over the entire barrel length, the feeding rate is fixed at $1 \mathrm{~kg} / \mathrm{h}$ and the screw rate is set at $300 \mathrm{rpm}$. The five temperature zones are set at 100, 130, 170, 180, and185C from feeder to die. The extruders are collect after cooling at ambient temperature on a conveyer belt. Samples are milled for $1 \mathrm{~min}$ with a laboratory cutting mill and sieve to exclude particles $>355 \mu \mathrm{m}$.

\section{Lyophilization technique $[11,12]$}

Lyophilization involves the transfer of heat and mass to and from the product under preparation. This technique was proposed as an alternative technique to solvent evaporation. Lyophilization has been thought of a molecular mixing technique where the drug and carrier are co-dissolved in a common solvent, frozen and sublimed to obtain a lyophilized molecular dispersion.

\section{Spray freeze drying}

Dissolve the drug in a solvent at a fixed concentration and carrier in water. Mix the solution in a ratio of $40 / 60 \mathrm{v} / \mathrm{v}$. spray the solutions through the nozzle into liquid nitrogen. Set the liquid feed rate and atomizing air flow. Position the outlet of the nozzle at about $10 \mathrm{~cm}$ above the liquid nitrogen. Hot water is pumped through the jacket of the nozzle in order to avoid freezing of the solution inside the nozzle. Transfer the resulting suspension (frozen droplets of the solution in liquid nitrogen) to the lyophilizer. Lyophilization procedure is started as soon as all liquid nitrogen is evaporated.

\section{Melt agglomeration process}

The utility of the surfactant systems insolubilization is very important. Adsorption of surfactant on the solid surface can modify their 
hydrophobicity, surface charge, and other key properties that govern interfacial processes such as flocculation/dispersion, floatation, wetting, solubilization, detergency, and enhanced oil recovery and corrosion inhibition. Surfactants have also been reported to cause solvation/plasticization, manifesting in the reduction of melting the active pharmaceutical ingredients, glass transition temperature and the combined glass transition temperature of solid dispersions. Because of these unique properties, surfactants have attracted the attention of investigators for the preparation of solid dispersions.

\section{Electrospinning}

Electrospinning is a process in which solid fibres are produced from a polymeric fluid stream solution or melt delivered through a millimeter-scale nozzle. This process involves the application of a strong electrostatic field over a conductive capillary attaching to a reservoir containing a polymer solution or melt and a conductive collection screen. Upon increasing the electrostatic field strength up to but not exceeding a critical value, charge species accumulated on the surface of a pendant drop destabilize the hemispherical shape into a conical shape (commonly known as Taylor scone). Beyond the critical value, a charged polymer jet is ejected from the apex of the cone (as a way of relieving the charge built-up on the surface of the pendant drop). The ejected charged jet is then carried to the collection screen via the electrostatic force. The Columbic repulsion force is responsible for the thinning of the charged jet during its trajectory to the collection screen. The thinning down of the charged jet is limited. If the viscosity increases, the charged jet is dried.

\section{Supercritical fluid (SCF) technology}

The supercritical fluid antisolvent techniques, carbon dioxide are used as and antisolvent for the solute but as a solvent with respect to the organic solvent. Different acronyms were used by various authors to denote micronization processes: aerosol solvent extraction system, precipitation with a compressed fluid antisolvent, gas anti-solvent, and solution enhanced dispersion by supercritical fluids and supercritical antisolvent. The SAS process involves the spraying of the solution composed of the solute and of the organic solvent into a continuous supercritical phase flowing concurrently. Use of supercritical carbon dioxide is advantageous as it is much easier to remove from the polymeric materials when the process is complete, even though a small amount of carbon dioxide remain strapped inside the polymer; it poses no danger to the patient. In addition, the ability of carbon dioxide to plasticize and swell polymers can also be exploited and the process can be carried out near the room. Moreover, supercritical fluids are used to lower the temperature of melt dispersion process by reducing the melting temperature of dispersed active agent. The reason for this depression is the solubility of the lighter component (dense gas) in the forming phase (heavier component).

\section{Selection of a carrier $[14,15]$}

A carrier should meet the following criteria to be suitable for increasing the dissolution rate of a drug.

- Freely water-soluble with intrinsic rapid dissolution properties.

- Non-toxic and pharmacologically inert.

- Heat stable with a low melting point for the melt method.

- Soluble in a variety of solvents and pass through a vitreous state upon solvent evaporation for the solvent method.

- Able to preferably increase the aqueous solubility of the drug.

- Chemically compatible with the drug and not form a strongly bonded complex with the drug

\begin{tabular}{ll}
\hline Carrier & Example \\
\hline First generation & Crystalline carriers: Urea, Sugars, Organic acids \\
Second generation & Fully synthetic polymers: PVP, PEG HPMC \\
Third generation & Surface active self-emulsifying carriers: Poloxamer 408, Tween 80, and Gelucire 44/141 \\
\hline
\end{tabular}

\section{Procedure of Solubilisation $[18,19]$}

Solubilisation process basically occurs in three steps:

\section{Holes open in a solvent}
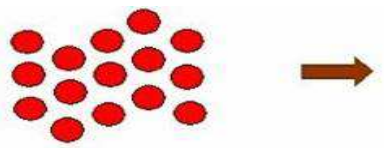

Molecules of the solid breaks away from the bulk
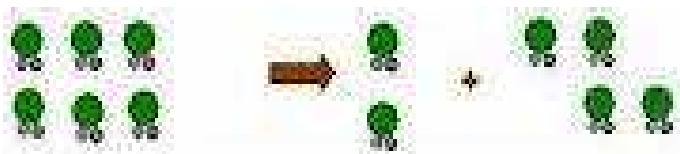

The freed solid molecule is integrated into the hole.

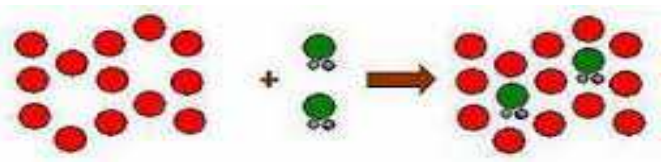

Factors affecting solubilisation [16, 17]

- Particle size

- Temperature

- Pressure

- Nature of solute and solvent

- Molecular size
- Polarity

- Polymorphs

\section{Advantages of solid dispersions}

Generally, solid dispersion is mainly used

- To reduced particle size.

- To improve wettability.

- To improve porosity of drug.

- To decrease the crystalline structure of drug into amorphous form.

- To improve dissolvability in water of a poorly water-soluble drug in a pharmaceutical.

- To mask the taste of the drug substance.

- To prepare rapid disintegration oral tablets.

- To obtain a homogeneous distribution of the small amount of drugs at solid state.

- To stabilize unstable drugs.

- To dispense liquid or gaseous compounds.

- To formulate a faster release priming dose in a sustained release dosage form.

- To formulate sustained release dosage or prolonged release regimens of soluble drugs using poorly soluble

\section{Applications of solid dispersions}

1. To increase the solubility of poorly soluble drugs thereby increase the dissolution rate, absorption and bioavailability. 
2. To stabilize unstable drugs against hydrolysis, oxidation, recrimination, isomerization, photo-oxidation and other decomposition procedures.

3. To reduce a side effect of certain drugs.

4. Masking of unpleasant taste and smell of drugs.

5. Improvement of drug release from ointment creams and gels.

6. To avoid undesirable incompatibilities.

7. To obtain a homogeneous distribution of a small amount of drug in solid state.

8. To dispense liquid (up to $10 \%$ ) or gaseous compounds in a solid dosage.

9. To formulate a fast release primary dose in a sustained released dosage form.

10. To formulate sustained release regimen of soluble drugs by using poorly soluble or insoluble carriers.

11. To reduce pre-systemic inactivation of drugs like morphine and progesterone

\section{Characterization of solid dispersion [13]}

- Phase solubility study.

- Production yield.

- Drug content.

- Dissolution study.

- Infra-red spectroscopy

- Powder X-ray diffraction.

- Differential scanning calorimetry.

\section{CONCLUSION}

Dissolution of the drug is the rate determining step for oral absorption of the poorly water soluble drug and solubility is the basic requirement for the absorption of the drug from GIT. The various techniques described above alone or in combination can be used to enhance the solubility of the drug. Proper selection of solubility enhancement method is the key to ensure the goals oral bioavailability, reduce the frequency of dosing and better patient compliance combined with a low cost of production. Selection of method for solubility enhancement depends upon drug characteristics like solubility, chemical nature, melting point, absorption site, physical nature, pharmacokinetic behavior and so forth, dosage form requirement like tablet or capsule formulation, strength, immediate, or modified release and so forth, and regulatory requirements like maximum daily dose of any excipients and drug, approved excipients, analytical accuracy.

\section{CONFLICT OF INTERESTS}

Declare none

\section{REFERENCES}

1. Lipinski CA. Avoiding investment in doomed drugs, is poor solubility an industry wide problem? Curr Drugs Dis 2001;4:17-9.
2. Sekiguchi K, Obi N. Studies on the absorption of eutectic mixture. I. A comparison of the behaviour of a eutectic mixture of sulfathiazole and that of ordinary sulfathiazole in man. Chem Pharm Bull 1961;9:866-72.

3. Goldberg AH, Gibaldi M, Kanig JL. Increasing dissolution rates and gastrointestinal absorption of drugs via solid solutions and eutectic mixtures II-experimental evaluation of a eutectic mixture: ureaacetaminophen system. J Pharma Sci 1966;55:482-7.

4. Castellan GW. Physical Chemistry, Addison-Wesley, Menlo Park, CA; 1983. p. 324-36.

5. Swarbrick J. ed 3. Encyclopaedia of pharmaceutical technology; 2006. p. 775-7.

6. Goldberg AH, Gibaldi M, Kanig JL. Increasing dissolution rates and gastrointestinal absorption of drugs via solid solutions and eutectic mixtures. I. Theoretical considerations and discussion of the literature. J Pharm Sci 1965;54:1145-8.

7. Hume Rotherly W, Raynor GV. The structure of metals and alloys, Institute of Metals, London; 1954.

8. Arunachalam A, Karthikeyan M, Konam K, Prasad P, Sethuraman S, Ashutoshraman S. Solid dispersions: a review, CPR; 2010. p. 82-90.

9. Patel PB, Prajapati ST, Patel NR, Patel NN, Patel CN. Solid dispersion: a method of improving bioavailability and taste masking. Inventi Rapid: Pharm Tech Vol 2013;3:427-39.

10. Ranade VV, Canon JB. Drug delivery systems. 3rd Edn. Published by Taylor and Francis group; 2011. p. 231-4.

11. Eun Jung Kim. Preparation of solid dispersion of felodipine using a solvent wetting method. Eur J Pharm Biopharm 2006;64:200-5.

12. X Wang, Michel A. Solid-state characteristics of ternary solid dispersions composed of PVP VA64, Myrj 52 and itraconazole. Int J Pharm 2005;303:54-61.

13. Leunar C, Dreessan J. Improving drug solubility for oral delivary using solid dispersion. Eur J Pharm Biopharm 2000;50:47-60.

14. OG Bhusnure, PA Kazi, SB Gholve, MMAW Ansari, SN Kazi. Review article solid dispersion: an evergreen method for solubility enhancement of poorly water soluble drugs. Int J Res Pharm Chem 2014;4:906-18.

15. PS Argade, DD Magar, RB Saudagar. Review article on solid dispersion as solubility enhancement technique for poorly water soluble drugs. J Adv Pharm Edu Res 2013;3:427-39.

16. Singh Jaskirat, Walia Manpreet, Harikumar SL. Formulation and evaluation of fast dissolving tablets of rosuvastatin. J Drug Delivery Ther 2013;3:148-55.

17. Neha Ojha, Bala Prabhakar. Advances in solubility enhancement techniques. Int J Pharm Sci Rev Res 2013;3:351-8.

18. Dixit AK, Singh RP, Singh Stuti. Formulation and evaluation of solid dispersion of amlodipine besylate. Int J Res Pharm Biomed Sci 2012;3:960-6.

19. Sameer Singh, Raviraj Singh Baghel, Lalit Yadav. A review on solid dispersion. Int J Pharm Life Sci 2014;2:1078-95.

20. Varun Raj Vemula, Venkateshwarlu Lagishetty, Srikanth Lingala. Combinational products: a regulatory review. Int J Pharm Sci Rev Res 2010;5:41-51.

21. Rajani Chauhan, Sneha Virmani, Suman. Quantitative method development and validation of sulfasalazine in tablet dosage form by uv-spectroscopy. Int J Pharm Sci Rev Res 2012;2:120-2.

\section{How to cite this article}

- AN Patil, DM Shinkar, RB Saudagar. Review article: solubility enhancement by solid dispersion. Int J Curr Pharm Res 2017;9(3):15-18. 